\title{
Experimental modal analysis of an electric locomotive body
}

\author{
Ion Manea ${ }^{1, *}$, Ioan Sebesan ${ }^{2}$, Gheorghe Ghita ${ }^{3}$, Mihai Matache ${ }^{4}$, Sorin Arsene ${ }^{2}$, and \\ Gabriel Prenta ${ }^{1}$ \\ ${ }^{1}$ SC Softronic SRL, R\&D Department, Craiova, No.40, Dolj, Romania \\ ${ }^{2}$ Polytechnic University of Bucharest, Sector 6, Splaiul Independentei, No. 313, Romania \\ ${ }^{3}$ Institute of Solid Mechanics of Romanian Academy, Bucharest, Constantin Mille, No.15, Romania \\ ${ }^{4}$ INMA Bucharest, Sector 1, Blvd. Ion Ionescu de la Brad, No. 6, Romania
}

\begin{abstract}
The study of the structural dynamics is essential for understanding and assessing of any engineering product performance, knowledge of dynamic structural response being fundamental to ensuring a sustainable and safe product functioning. From all techniques for structural response assessment, the experimental modal analysis (EMA) provides one of the most comprehensive tools for collecting the experimental data, relevant for structure investigation and assessing of the structural response under normal or imposed operating conditions. Once identified the structure in terms of modal model, it can begin to its optimization to face new challenges or functioning tasks. The article presents an experimental modal analysis application, performed on a LEMA $6000 \mathrm{~kW}$ electric locomotive body on the production cycle of SC Softronic Craiova. The application was made to validate the dynamic finite elements model realised in order to certify the crashworthiness locomotive design and to evaluate the body interaction with bogie and track to avoid the locomotive structural resonance phenomena.
\end{abstract}

\section{Background of experimental modal analysis}

Experimental modal analysis (EMA) is the process of building the mathematical model of a system based on experimental determined modal parameters in view to investigate the structural response to the given load conditions, to evaluate the effect of controlled structural changes, or to evaluate the structure response to the required conditions in new applications.

Usually, by using the numerical analysis techniques it is done the analysis of structure vibration state in terms of frequency response function (FRF). A set of FRF measurements carried out in well-defined conditions and points on the structure, can provide a comprehensive response of the structure to specified conditions of excitation.

Through experimental modal analysis are determined the modal parameters of the system in terms of: modal frequencies, modal damping and modal shapes, which together provide a

\footnotetext{
${ }^{*}$ Corresponding author: ion.manea@softronic.ro
} 
complete description of the intrinsic dynamic characteristics of the structure, independent of the structural excitation.

Modal analysis can be achieved through both analytical techniques and experimental techniques, the two types of analysis leading practically to the same modal parameters. A complete modal analysis assumes utilization of the combined techniques, analytical and experimental, in this case the experimental model serves as a validation tool of analytical model. Modal models are not the models of the system itself, but rather are models of dynamic behavioural of the system constrained by a set of hypotheses and boundary conditions.

The analytical model is based on equations associated with the system mass and stiffness distribution, calculated by imposing a given set of boundary conditions. The analytical model, usually, is prepared using the finite elements method (FEM) and associated mathematical model involves a set of complicated coupled differential equations that can be solved only by using advanced computing techniques.

The experimental model is based on vibrational response data measured on the system in well-defined conditions, which is a mirror of the system under measurement. Associated mathematical model consists of a limited set of differential equations, one for each degree of freedom. This model is often referred as "experimental modal model".

Experimental modal analysis techniques are used in a wide range of applications, including: structural optimization; prediction of dynamic behaviour; assessment of the excitation forces from only vibration response; prediction of structural response under the effect of complex excitations; validation of analytical finite elements model by experimental data; detection, location and evaluation of structural defects.

\section{Mathematical models used in experimental modal analysis}

The most general mathematical model is one in which the matrices of mass, stiffness and damping forces are estimated based on measurements of vibrational excitation and response.

The mathematical model is based on a system of differential equations adapted to the calculation method, which can be in time domain or frequency domain. Equation (1) shows the general form of the moving equation for the time domain, and equation (2) shows the general form of the moving equation for the frequency domain.

$$
\begin{gathered}
{[M]\{\ddot{x}(t)\}+[C] \cdot\{\dot{x}(t)\}+[K]\{x(t)\}=\{f(t)\}} \\
-\omega^{2}[M]\{X(\omega)\}+i \omega[C] \cdot\{X(\omega)\}+[K]\{X(\omega)\}=\{Q(\omega)\}
\end{gathered}
$$

If the equations (1) and (2) are used as a model to estimate the modal parameters, the unknown matrices elements must first be estimated from experimental data, through measurements carried out in time or frequency domains. Once the matrices elements were estimated, the modal parameters are found as solution of a eigenvalues and eigenvectors problem.

Because of the truncated data in terms of content, frequency range, number of degrees of freedom, or due to errors in measurements, the matrices in the equations (1) and (2) are not generally directly comparable with the matrices determined by an finite element approach. Instead, the matrices which are estimated by such procedures provide valid relationships between input-output quantities and correct modal parameters. This is because there are an infinite number of sets of matrices of mass, damping and stiffness which leads to the same modal parameters in the same frequency range and in the same measurement range. The most mathematical models describing the dynamics of an elastic system with $\mathrm{N}$ degrees of freedom, can be arranged in a suitable form, in such a way as to be described by the following relationships defined in the time domain (relation 3) or frequency domain (relation 4) : 


$$
\begin{gathered}
h_{p q}(t)=\sum_{r=1}^{N} A_{p q r} e^{\lambda_{r} t}+A_{p q r}^{*} e^{\lambda_{r}^{*} t} \\
H_{p q}(\omega)=\sum_{r=1}^{N} \frac{A_{p q r}}{i \omega-\lambda_{r}}+\frac{A_{p q r}^{*}}{i \omega-\lambda_{r}^{*}} \\
A_{p q r}=Q_{r} \Psi_{p r} \Psi_{q r} \\
A_{p q r}=\Psi_{p r} \mathrm{~L}_{q r}
\end{gathered}
$$

In relations (1) ... (6) have been used the following notations [1]: $\omega$ - angular frequency variable ( $\mathrm{rad} / \mathrm{sec}) ; \mathrm{p}$ - measured degree-of-freedom (response); $\mathrm{q}$ measured degree-of-freedom (input); $\mathrm{r}$ - modal vector number; $\mathrm{N}$ - number of modal frequencies; $A_{p q r}$ - residue for mode r; $Q_{r}$ - complex modal scaling coefficient for mode $\mathrm{r}$; $\Psi_{p r^{-}}$modal coefficient for measured degree-of-freedom $\mathrm{p}$ and mode $\mathrm{r} ; \mathrm{L}_{q r^{-}}$modal participation factor for reference degree-of-freedom $\mathrm{q}$ and mode $\mathrm{r} ; \lambda_{r}-$ system pole.

The great majority of modal parameter estimation techniques assume that the system is linear and time invariable. In fact, depending on the analysed system and on conditions under which excitation was carried out, these assumptions are valid in a proportion greater than or less than, but almost never true at all. The analytical model represented by equations (4) is a nonlinear model in terms of modal parameters, $\lambda_{r}$. The difference between the various modal parameter estimation algorithms consists in the modality of solving the nonlinearities of mathematical models. There are a wide variety of algorithms for determining the modal parameters, but the most used techniques are: Rational Fraction Polynomial-Z (RFP-Z) and Polyreference Time (PDT). Both techniques works with complex modes and have the great advantage that can process data input from multi-reference imputes. In this way, any situations of real modes or unireference excitation can be treated as a particular case.

\section{Experimental modal analysis applications to railway vehicles}

\subsection{Excitation and measuring equipment and software}

-Piezoelectric accelerometers type 355B03, 15 pieces, sensitivity: $10.19 \mathrm{mV} /\left(\mathrm{m} / \mathrm{s}^{2}\right)$; measuring range: $\pm 490 \mathrm{~m} / \mathrm{s}^{2}$; frequency range: $2-5000 \mathrm{~Hz}$;

-Force transducer type $208 \mathrm{C} 03$, sensitivity: $2248 \mathrm{mV} / \mathrm{kN}$; measuring range: $\pm 2.224 \mathrm{kN}$; frequency range: $36 \mathrm{kHz}$;

-Electrodynamic shaker type 11077 , force range: $\pm 100 \mathrm{~N}$, frequency range: $3 \mathrm{~Hz} \div 20 \mathrm{kHz}$;

-Power amplifier type LV103, power: 100VA, frequency range: $3 \mathrm{~Hz} \div 20 \mathrm{kHz}$;

-Data acquisition system type SoftronicDataAcquisition, carrying case including 1xLANXI type 3160A042 and 1xLAN-XI type 3053B120, with the following main characteristics: inputs: 16(voltage and CCLD/ICP); outputs: 2 (voltage outputs from generator); energy independence with all acquisition systems in operation: 5 hours;

-PULSE LabShop, software module for data acquisition control and real-time multichannel processing;

-Structural Dynamic Test Consultants, software module working under PULSE LabShop, providing a dedicated environment for modal analysis tests driving using the classical excitation technics of impact hammer or shaker. Drives the measurements through simple steps, providing an intuitive graphical interface which connects the test configuration, geometry of test structure and information concerning the degrees of freedom of excitation and response. At the final stage, the measured FRF together with the structure geometry are transmitted to PULSE Reflex Modal Analysis, which is automatically launched.

-PULSE Reflex Modal Analysis, post-processing mode for classical modal analysis. Uses a wide variety of modal identification modules, including PTD and RFP techniques. 
-PULSE Reflex Correlation Analysis, correlation analysis module that provides the verification and validation of analytical models obtained by finite element methods through the experimental modal analysis tests.

\subsection{Experimental modal analysis test on an electric locomotive body}

During the tests the locomotive body was only equipped with the two obstacle deflectors, without caps doors and other equipment. To ensure a correct application of EMA techniques, it was ensuring the conditions of pseudo-freedom vibrations of the body by suspending it on four winches, which, by their lifting axle and sole support, provides enough elasticity to consider the locomotive body as a free body, for the low level vibration displacements, so not restricting the degrees of freedom of the body [2].

It has used the method of excitation in one point by sinusoidal excitation sweep in the frequency range of 2-200 Hz with electrodynamic shaker attached to the strength structure of sideframe, under the driver's cab, near the mounting point of obstacle deflector. The excitation force transmission was carried out by means of a spring steel rod with a diameter of $5 \mathrm{~mm}$, to avoid the effects of parasitic coupling between the shaker and body. The mounting schemes for EMA tests is presented in Fig. 1.

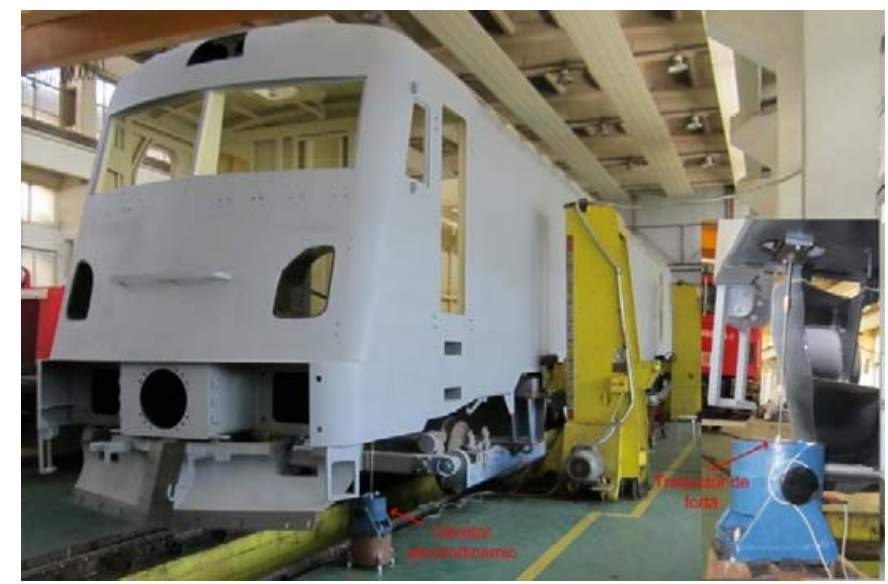

Fig. 1. Experimental modal analysis test on a locomotive body using the shaker excitation method.

Data acquisition was carried out under SDTC in which it was achieved the body geometry and it has been developed the test program, being planned eight sequences for measuring of dynamic response. For the 8 measuring sequences, the structure excitation was maintained at said point, point 67 as shown in Figure 2. At one measuring sequence, the dynamic response was determined in 5 measuring points, for each point being determined the vibratory response on the vertical, longitudinal (along the body) and transverse (across the body) directions. So for each sequences were measured 15 degree of freedom of response and 1 degree of freedom of reference (excitation). The global EMA test involved a total of 120 measuring response for one reference point 67, resulting in a total of 120 frequency response functions.

Figure 2 presents the panel driving for one measuring sequences under SDTC. On the geometric model it is positioned the shaker excitation in 67 point and frequency response functions of points $36,49,75,46$ and 53 in the $7^{\text {th }}$ measuring sequence. The distribution of the measurement points was carried out in such a way as to be representative for dynamic response of locomotive body and to be enough distanced in order to achieve a correct body animation in the natural vibration modes. To achieve a high accuracy of FRF, has selected a frequency resolution of $62.5 \mathrm{mHz}$ and a linear mediation over $237 \mathrm{FRF}$ sequences. 


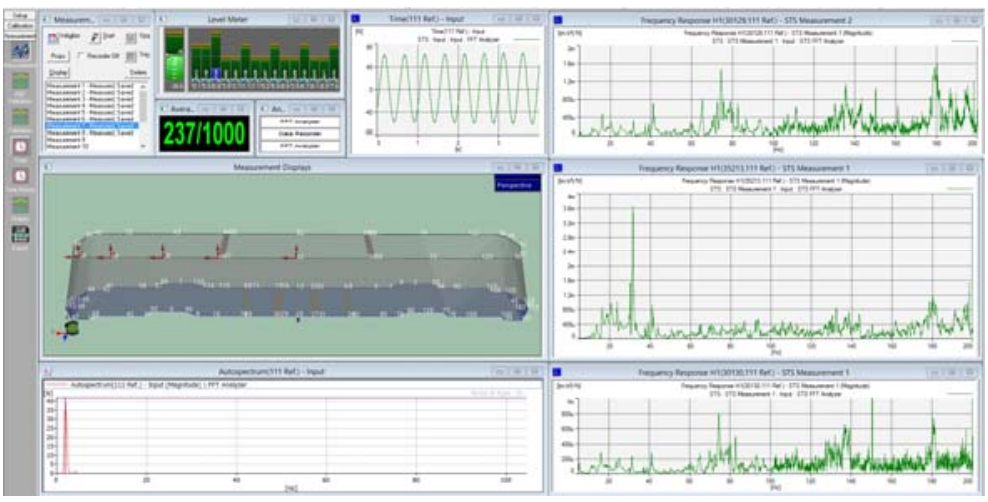

Fig. 2. The driving panel of EMA tests under Structural Dynamic Test Consultants.

\subsection{Modal analysis of electric locomotive body}

Modal analysis of recorded data was performed with PULSE Reflex Modal Analysis. In the first stage it was done the validation of acquired data by analysing the noise in excitation and response, as well as by structure animation at different frequencies in the test domain, as in Figure 3. In this step can be eliminated the aberrant data.

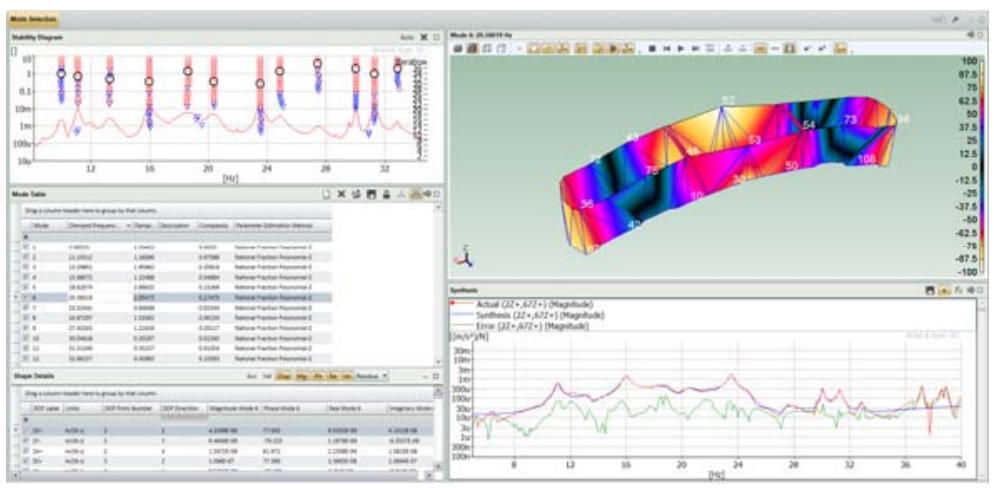

Fig. 3. The validation panel of PULSE Reflex Modal Analysis.

In this paper have been used two methods to estimate the modal parameters, Rational Fraction Polynomial-Z (RFP-Z) and Polyreference Time (PDT), both the higher order methods, recommended for working in wide band frequency, being able to working with large number of modes. Given the purpose application, validating the analytical model with finite elements, the frequency domain was limited to $5 \ldots 35 \mathrm{~Hz}$.

Figures $4 \ldots 11$ presents the modal shapes of locomotive body in their first 8 vibration eigenmodes in frequency domain of $5 \ldots 35 \mathrm{~Hz}$.

Modal shapes can be exported in a tabular form providing: damped frequency $(\mathrm{Hz})$, damping (\%), and mode complexity. For each of modes are transmitted separately for each measured degree of freedom (DOF) the following parameters needed for the modes rebuilding: DOF direction, mode magnitude, mode phase. These parameters are transmitted separately for: residue, unity vector length and unity modal mass.

Mode shapes determined by EMA serve as a validation tool for analytical model realised by finite elements analysis. Validated FEA model was used for evaluating the collisions resistance of LEMA locomotive and to estimate the body interaction with the bogie and track to avoid the structural resonance phenomena. This analysis are not the subject of this article. 


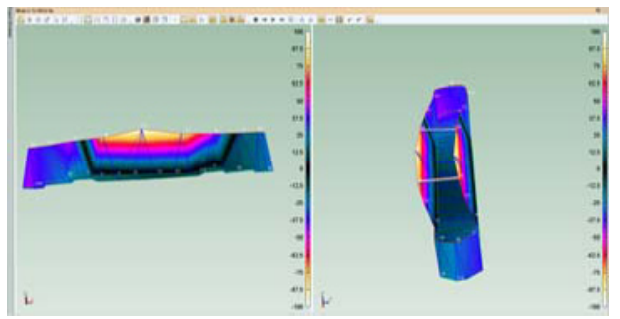

Fig. 4. The $1^{\text {st }}$ vibration mode, $\mathrm{Fq}=11.10 \mathrm{~Hz}$

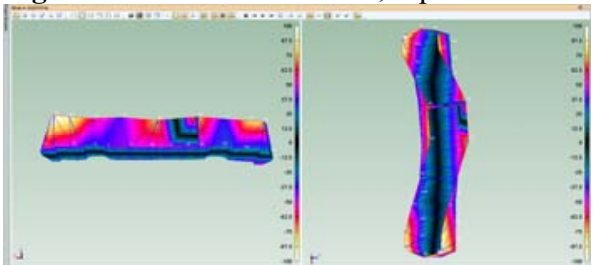

Fig. 6. The $3^{\text {rd }}$ vibration mode, $\mathrm{Fq}=18.62 \mathrm{~Hz}$

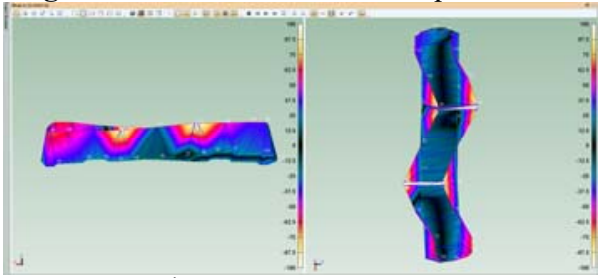

Fig. 8. The $5^{\text {th }}$ vibration mode, $\mathrm{Fq}=23.52 \mathrm{~Hz}$

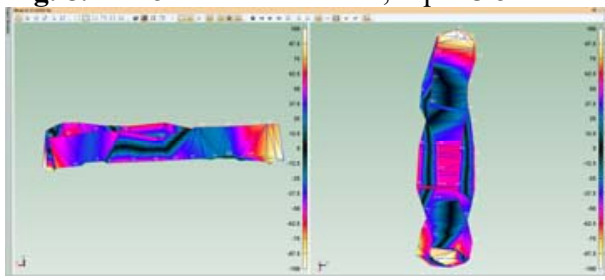

Fig. 10. The $7^{\text {th }}$ vibration mode, $\mathrm{Fq}=27.43 \mathrm{~Hz}$

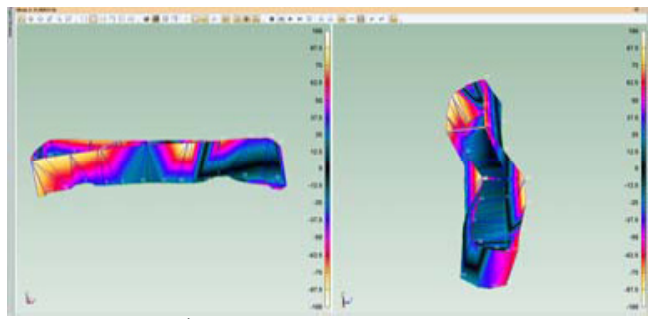

Fig. 5. The $2^{\text {nd }}$ vibration mode, $\mathrm{Fq}=15.98 \mathrm{~Hz}$

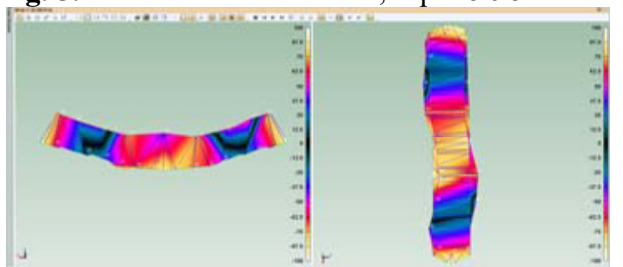

Fig. 7. The $4^{\text {th }}$ vibration mode, $\mathrm{Fq}=20.38 \mathrm{~Hz}$

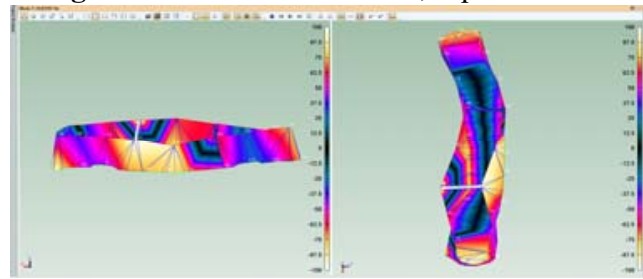

Fig. 9. The $6^{\text {th }}$ vibration mode, $\mathrm{Fq}=24.87 \mathrm{~Hz}$

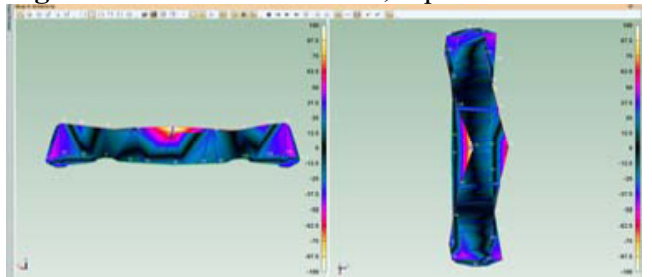

Fig. 11. The $8^{\text {th }}$ vibration mode, $\mathrm{Fq}=30.04 \mathrm{~Hz}$

\section{Conclusions}

The article presents an EMA application performed on an empty locomotive body in order to achieve the modal model which shall be later used as a validation tool for analytical finite element model. The modal model is described through: damped frequency $(\mathrm{Hz})$, damping $(\%)$, and mode complexity. It can be successfully used for prediction of the body structural response under the effect of complex excitations like as that from bogie and track.

This work was supported by a grant of the Romanian National Authority for Scientific Research and Innovation, CNCS-UEFISCDI, project number PN-III-P2-2.1-PTE-2016-0008, within PNCDI III

\section{References}

1. R. J. Allemang, D. L. Brown, Experimental Modal Analysis and Dynamic Component Synthesis - Modal Parameter Estimation, vol3 (1987)

2. I Manea, I Sebesan, Gh. Ghită, Modal analysis of theoretical and experimental. Application to railway vehicles, Bucharest, Matrix (2016) 